\title{
Evaluación y seguimiento del Aprendizaje Permanente
}

\author{
Debón-Aucejo, Ana María ${ }^{a}$; García-Gallego, Carlos Vicente ${ }^{\text {a }}$; Hervas-Oliver, Jose \\ Luis $^{\text {a }}$ y Pérez-Yuste, Maria Soledad ${ }^{a}$
}

aFacultad de Administración y Dirección de Empresas (Universitat Politècnica de València), andeau@eio.upv.es, cargarga@urb.upv.es, jose.hervas@omp.upv.es, mapeyus@esp.upv.es.

\begin{abstract}
The teaching and evaluation of transversal competences in the university environment is complex and they should be specified in learning outcomes in specific subjects at specific levels. These competences are skills related to personal development that do not depend on a specific thematic area but that do appear in all domains of professional and academic performance. Therefore, employers do not hesitate to emphasize the great importance it has in the professional performance of university graduates.

So the Universitat Politècnica de València (UPV) has launched an ambitious institutional project certification to the student of their training in skills. In this paper, we take care to describe the framework that has been given to the transversal competence 11 "Lifelong learning" in the Facultad de Administración y Dirección de Empresas (FADE) of the UPV, as well as the methodologies used for its evaluation in the subjects used as a control point on two different levels.
\end{abstract}

Keywords: Lifelong learning, transversal skills, ADE degree.

\section{Resumen}

La enseñanza y evaluación de las competencias transversales en el ámbito universitario es compleja y se debe concretar en resultados de aprendizaje en materias determinadas en niveles especificos. Estas competencias son habilidades relacionadas con el desarrollo personal que no dependen de un ámbito temático especifico pero que si aparecen en todos los dominios de la actuación profesional y académica. Por ello, los empleadores no dudan en subrayar la gran importancia que tiene en el desempeño profesional de los titulados universitarios.

Así pues, la Universitat Politècnica de València (UPV) ha puesto en marcha un ambicioso proyecto institucional certificación al estudiante de su formación en competencias. En este trabajo nos encargamos de describir el marco que se ha dado a la competencia transversal 11 "Aprendizaje permanente” en la Facultad de Administración y Dirección de Empresas (FADE) de la UPV, asi como las metodologías utilizadas para su evaluación en las asignaturas utilizadas como punto de control en dos niveles diferentes.

Palabras clave: Aprendizaje permanente, competencias transversales, grado de $A D E$.

Esta obra está bajo una Licencia Creative Commons CC BY-NC-ND 4.0

EDITORIAL UNIVERSITAT POLITÈCNICA DE VALÈNCIA 


\section{Introducción}

Actualmente ha cobrado relevancia la enseñanza y evaluación de las competencias transversales en el ámbito universitario y aunque los profesores nos seguimos preguntado que son, los graduados y empleadores no dudan en subrayar la gran importancia que tiene en el desempeño profesional de los titulados universitarios. De acuerdo a González y Wagenaar (2003) las competencias transversales son aquellas habilidades relacionadas con el desarrollo personal, que no dependen de un ámbito temático o disciplinario especifico sino que aparecen en todos los dominios de la actuación profesional y académica.

Así pues y dado que se trata de algo complejo y general debemos de concretarlo en resultados de aprendizaje más específicos en determinados niveles y materias de los títulos de grado.

Según el proyecto institucional sobre competencias transversales de la Universitat Politècnica de Valencia (UPV)(2015) éste representa un valor añadido para sus titulados, además de una mejora continua de los títulos y posibilita el acceso de la universidad a acreditaciones tanto nacionales como internacionales. El proyecto es muy ambicioso pues declara como intenciones la certificación al estudiante de su formación en competencias y así hacer conocedor al empleador del nivel adquirido por el egresado.

En este trabajo nos encargamos de describir el marco que se ha dado a la competencia transversal 11 "Aprendizaje permanente" en la Facultad de Administración y Dirección de Empresas (FADE) de la UPV, así como las metodologías utilizadas para su evaluación en las asignaturas utilizadas como punto de control en dos niveles diferentes.

\section{Contexto}

La competencia transversal 11 se define como "Utilizar el aprendizaje de manera estratégica, autónoma y flexible, a lo largo de toda la vida, en función del objetivo perseguido" (UPV, 2019).

Para evaluar dicha competencia en el grado en Administración y Dirección de Empresas de FADE se han determinado como puntos de control 4 asignaturas de acuerdo a la Tabla 2 .

Tabla 2. Asignaturas punto de control

\begin{tabular}{ccc}
\hline Asignatura & Curso & Nivel \\
\hline $\begin{array}{c}\text { Derecho del } \\
\text { Trabajo }\end{array}$ & $2^{\mathrm{o}}$ & 1 \\
Estrategia y & & \\
$\begin{array}{c}\text { Diseño de la } \\
\text { Organización }\end{array}$ & $2^{\mathrm{o}}$ & 1
\end{tabular}




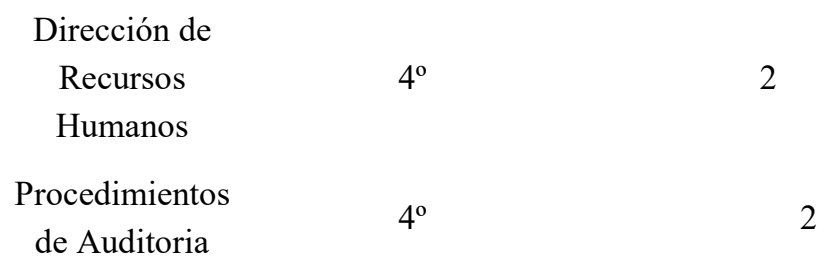

Las dos asignaturas de $2^{\circ}$ curso del grado de ADE constituyen la evaluación de la competencia en nivel 1 mientras que las dos de $4^{\circ}$ constituyen el nivel 2 , el tercer nivel se evalúa ya en master y no es objeto de este trabajo.

Con ello se persigue tener un seguimiento de la evolución del estudiante en la competencia.

\subsection{Metodologías de evaluación}

Las metodologías utilizadas en las diferentes asignaturas son muy variadas, a continuación, pasamos a describirlas.

\subsubsection{Estrategia y Diseño de la Organización}

Según se señala en la Tabla 2 la asignatura Estrategias y Diseño de la organización se imparte en 2 curso del grado de ADE por el profesor Jose Luis Hervas Oliver uno de los autores de este trabajo. Es una de las primeras asignaturas que trabaja la competencia por lo que se ha diseñado una prueba lo más amena posible y de contacto con la realidad.

Durante todo el semestre se enfatiza la búsqueda activa de noticias y su análisis crítico para la evaluación de la competencia.

\subsubsection{Derecho del Trabajo}

De nuevo se vuelve a evaluar la competencia en la asignatura Derecho del Trabajo (Tabla 2) impartida en $2^{\circ}$ curso por Carlos Garcia Gallego autor también de este trabajo. La evaluación de esta competencia en la asignatura Derecho del Trabajo, de segundo curso del grado en ADE, se establece en dos etapas:

Por un lado, mediante el estudio de casos. En una sesión de clase, se expone un caso complejo en el que los alumnos, por grupos reducidos, deben dar solución al problema planteado.

Este caso, en ocasiones, está basado en hechos reales (por ejemplo, cuando se trata de supuestos basados en sentencias de los tribunales) y en otras ocasiones, se trata de supuestos ficticios pero que perfectamente pueden darse en la realidad empresarial.

El caso que se entrega a los alumnos se caracteriza por no tener una respuesta cerrada, sino que pueden aplicarse diferentes soluciones, todas ellas perfectamente válidas, pero con consecuencias jurídicas diferentes para la empresa. De este modo, no sólo se trata de entender el supuesto de hecho a partir de los conocimientos que ya tiene el alumno, sino que además debe buscar una solución al problema planteado y calcular las diferentes consecuencias o efectos que la misma pudiera tener para la empresa. 
Una vez determinadas las posibles soluciones del caso planteado, cada grupo debe exponerla delante del resto de la clase para finalmente, debatir entre todas las diferentes soluciones aportadas.

Un ejemplo de estudio de caso en la asignatura Derecho del Trabajo sería plantear un supuesto, en el cual una empresa atraviesa por una crisis económica y, en función de las circunstancias concretas que se planteen, el alumno debe tomar, respecto de la plantilla de trabajadores, las decisiones que estime más adecuadas para revertir la situación (por ejemplo, si conviene o no despedir a trabajadores, cuánto costaría, qué consecuencias tendría sobre el resto de trabajadores, si puede o no adoptarse alguna medida alternativa al despido, qué consecuencias tendría esto entonces....).

Junto con el estudio de casos, se plantea también la evaluación de esta competencia mediante visitas externas. Concretamente mediante la asistencia a uno o varios juicios laborales en los juzgados de lo social de Valencia.

En esta visita los alumnos, en grupos reducidos asisten (al menos) a un juicio laboral de modo que entran en contacto directo con la realidad de la materia que se imparte en clase.

Una vez los alumnos han asistido al juicio correspondiente se debate en clase sobre el contenido del mismo y deben aportar posibles soluciones al supuesto planteado en el mismo. En otras palabras, deben dictar ellos la sentencia que crean oportuna (conviene recordar que al finalizar el juicio el juez no dicta la sentencia de forma inmediata de modo que los alumnos desconocen el contenido de la misma).

Cada grupo expone delante del resto de la clase el contenido del juicio al que ha asistido y propone la correspondiente sentencia. Al finalizar la exposición el resto de la clase puede plantear las cuestiones que estime convenientes.

\subsubsection{Procedimientos de auditoria}

En $4^{\circ}$ curso del grado de ADE se evalúa una vez más la competencia en la asignatura Procedimientos en Auditoria que imparte $\mathrm{M}^{\mathrm{a}}$ Soledad Pérez Yuste autora de este trabajo.

En este nivel 2 se utiliza una metodología más de aplicación en supuestos prácticos. En las sesiones de prácticas, se solucionan supuestos prácticos de auditoría en los que se analiza la aplicación de la normativa de auditoría y las normas contables: planes de contabilidad, resoluciones y consultas del Instituto de Contabilidad y Auditoría de Cuentas (ICAC), normas internacionales de contabilidad, normas internaciones de auditoría, Normas Técnicas de Auditoría resultado de la adaptación de las Normas Internacionales de Auditoría para su aplicación en España (NIA-ES), Normas Internacionales de las Entidades Fiscalizadoras Superiores desarrolladas por la Organización Internacional de Entidades Fiscalizadoras Superiores (INTOSAI) adaptadas a España (ISSAI-ES), etc.

Se plantean supuestos reales en los cuales un auditor realiza la auditoría financiera de una empresa, analizando la razonabilidad de los datos incluidos en las cuentas anuales. Para ello plantea sus procedimientos de auditoría y obtiene evidencias de auditoría. 
En base a los resultados obtenidos, el alumno debe aplicar la normativa de auditoría a fin de elaborar sus conclusiones y emitir el pertinente informe de la misma. Dicho informe debe adaptarse a la legalidad vigente (ley de auditoría, reglamento de auditoría, NIA-ES, legislación mercantil y resto de la normativa aplicable).

El alumno podría acceder mediante internet, en los ordenadores del laboratorio, a distintas fuentes de información y recursos disponibles a fin de aplicar correctamente el derecho contable y la normativa reguladora de la auditoria en España. En base a las consultas efectuadas realizará el supuesto de auditoría planteado en la práctica. La competencia transversal se evaluará mediante rúbrica a aplicar en la evaluación de la práctica.

La práctica se realiza por parejas y se corregirá de forma anónima, por lo que se le asigna a cada pareja un número de grupo.

El alumno debe aplicar los conocimientos teóricos obtenidos en la asignatura, analizar las evidencias de auditoría del supuesto, buscar la normativa de auditoría y contable aplicable al caso, revisar las resoluciones del ICAC, indagar las soluciones posibles, investigar las soluciones profesionales emitidas por el ICAC y por las Corporaciones representativas de auditores de cuentas y elaborar las conclusiones.

Finalmente debe elegir los párrafos pertinentes del informe de auditor en función de una gama amplia de párrafos estándar incluidos en las NIA-Es y adaptarlos al caso analizado, ya que el formato y la terminología del informe de auditoría están regulados legalmente.

En auditoría es muy importante la supervisión del trabajo realizado, para poner en práctica dicho principio el profesor fotocopia los ejercicios realizados y los entrega para su revisión, análisis, comentario y evaluación a alumnos de grupo distinto.

La nota de la práctica de cada alumno tiene dos componentes: la solución del supuesto práctico y la revisión efectuada de la solución dada por otro grupo.

Se reproduce así la realidad de una auditoría financiera apoyándonos en un caso los más real posible basado en la experiencia del profesorado.

\subsection{Ventajas e Inconvenientes de las metodologías}

En cuanto a las ventajas, creemos que la metodología escogida para la evaluación de la competencia obliga al alumno a tener una visión global de las asignaturas y a darse cuenta del funcionamiento de los contenidos de la asignatura en supuestos reales, en particular en lo que se refiere a la visita al juicio en Derecho del trabajo. El alumno por su parte agradece que se planteen retos tan próximos a la realidad empresarial de la que en principio deben formar parte una vez se gradúen.

También cabe destacar que los supuestos están basados en la experiencia profesional de los profesores asociados, como es el caso de $\mathrm{M}^{\mathrm{a}}$ Soledad en Procedimientos de auditoria, y de esta forma puede aportar a los estudiantes todo lo aprendido en el mundo laboral

En cuanto a los inconvenientes, básicamente se han planteado dos, en el caso de derecho del trabajo. Por una parte, los juicios que pueden verse en una mañana son muy variados 
y se han dado situaciones en las que los alumnos no han podido ver ninguno que resulte de verdad interesante. Además, la organización de la visita no es fácil puesto que no todos los días hay juicios, solo hay por las mañanas, hay que pedirles a otros profesores que cedan sus sesiones de clase para poder organizar la visita lo que no siempre es fácil ni posible. Por otra parte, en ocasiones los contenidos de las sesiones de juicio son demasiado complejos para alumnos que no tienen una formación jurídica sólida.

En cuanto a procedimientos de auditoria la desventaja fundamental está en la falta de control del trabajo individual y la trasferencia de conocimiento que se produce de unos grupos a otros por el ordenador y otros canales.

Finalmente, y como soluciones a estas desventajas se plantea la posible competición entre los grupos para fomentar su colaboración dentro del grupo, pero no fuera de él.

Y también la corrección por pares de los trabajos utilizando la herramienta del PoliformaT. PoliformaT es el campus virtual de la Universidad Politécnica de Valencia (https://poliformat.upv.es/portal). Pero también es una nueva plataforma de e-learning que ofrece una amplia variedad de herramientas de apoyo a la docencia presencial y online. En este sentido, la mayoría de las instituciones universitarias están apostando por el uso de plataformas de teleformación o campus virtuales, como una vía para poder realizar prácticas educativas venciendo las limitaciones espacio-temporales (Muñoz-Carril y Gonzalez-Sanmamed, 2009).

\section{Conclusiones}

En primer lugar, cabe destacar que en este trabajo hemos descrito el marco que se ha dado a la competencia transversal 11 "Aprendizaje permanente" en la Facultad de Administración y Dirección de Empresas (FADE) de la UPV, así como algunas de las metodologías utilizadas para su evaluación en las asignaturas utilizadas como punto de control en dos niveles diferentes.

En segundo lugar, hay que señalar que dichas metodologías se adaptan a cada asignatura y nivel, aprovechan la experiencia profesional de los docentes y ponen en contacto a los estudiantes con la práctica profesional.

Además, y de acuerdo al proyecto institucional sobre competencias transversales de la UPV, esto supone que se aborda metodología para la certificación al estudiante de su formación en competencias que representa un valor añadido para los titulados. De esta forma, además de una mejora continua de los títulos se posibilita el acceso de la universidad a acreditaciones tanto nacionales como internacionales, pero también hace conocedor al empleador del nivel adquirido por el egresado.

Finalmente, se plantean desventajas de las metodologías y posibles acciones para superarlas. Dichas acciones son la posible competición entre grupos o el uso de herramientas de PoliformaT. 


\section{Referencias}

GONZALEZ, J., \& WAGENAAR, R. (Eds.). (2003). Tuning educational structures in Europe. Bilbao: University of Deusto.

MUÑOZ-CARRIL, P.C. y GONZALEZ-SANMAMED, M. G. (2009). Plataformas de teleformación y herramientas telemáticas. Editorial UOC.

UNIVERSITAT POLITÈCNICA DE VALÉNCIA (2015). Competencias transversales. $<$ https://www.upv.es/entidades/ICE/info/Proyecto_Institucional_CT.pdf $>$ [Consulta: 25 de mayo de 2019].

UNIVERSITAT POLITÈCNICA DE VALÉNCIA (2019). Competencias transversales. $<$ http://www.upv.es/contenidos/COMPTRAN/info/955154normalc.html [Consulta: 25 de mayo de 2019]. 